Keywords: male pattern baldness; frontal-only baldness; frontal-plus-mild-vertex baldness; frontal-plus-moderate-vertex baldness; frontal-plus-severe-vertex baldness; colorectal cancer; colorectal adenoma; colorectal neoplasia

\title{
Male pattern baldness and risk of colorectal neoplasia
}

\author{
N Keum ${ }^{*}, 1,2$, Y Cao ${ }^{1,2}$, D H Lee ${ }^{1,2}$, S M Park ${ }^{3}$, B Rosner ${ }^{4}$, C S Fuchs ${ }^{5}$, K Wu ${ }^{1,8}$ and E L Giovannucci ${ }^{1,2,6,7,8}$ \\ ${ }^{1}$ Department of Nutrition, Harvard T.H. Chan School of Public Health, Building 2, 3rd Floor, 665 Huntington Avenue, Boston, MA \\ 02115, USA; ${ }^{2}$ Department of Epidemiology, Harvard T.H. Chan School of Public Health, Building 2, 3rd Floor, 665 Huntington \\ Avenue, Boston, MA 02115, USA; ${ }^{3}$ Department of Family Medicine, Seoul National University Hospital, Seoul National University \\ College of Medicine, Seoul, Korea; ${ }^{4}$ Department of Biostatistics, Harvard T.H. Chan School of Public Health, Building 2, 3rd Floor, \\ 665 Huntington Avenue, Boston, MA 02115, USA; ${ }^{5}$ Department of Oncology, Dana-Farber Cancer Institute, Boston, MA 02215, \\ USA; ${ }^{6}$ Department of Medicine, Harvard Medical School, Boston, MA 02115, USA and ${ }^{7}$ Channing Division of Network Medicine, \\ Brigham and Women's Hospital and Harvard Medical School, Boston, MA 02115, USA
}

Background: Male pattern baldness is positively associated with androgens as well as insulin-like growth factor 1 (IGF-1) and insulin, all of which are implicated in pathogenesis of colorectal neoplasia.

Methods: From 1992 through 2010, we prospectively followed participants in the Health Professionals Follow-Up Study. Hair pattern at age 45 years was assessed at baseline with five image categories (no baldness, frontal-only baldness, frontal-plus-mildvertex baldness, frontal-plus-moderate-vertex baldness, and frontal-plus-severe-vertex baldness). Cancer analysis included 32782 men and used Cox proportional hazards models to estimate hazard ratios (HRs) and 95\% confidence intervals (Cls). Restricted to men who underwent at least one endoscopy over the study period, adenoma analysis included 29770 men and used logistic regressions for clustered data to estimate odds ratios (ORs) and 95\% Cls.

Results: Over the mean follow-up of 15.6 years, 710 cases of colorectal cancer (478 for colon, 152 for rectum, and 80 unknown site) developed. Significantly increased risks associated with frontal-only baldness and frontal-plus-mild-vertex baldness relative to no baldness were observed for colon cancer with respective HR being 1.29 (95\% Cl, 1.03-1.62) and 1.31 (95\% Cl, 1.01-1.70). Over the 19 -year study period, 3526 cases of colorectal adenoma were detected. Evidence for an increased risk of colorectal adenoma relative to no baldness was significant with frontal-only baldness $(\mathrm{OR}, 1.16 ; 95 \% \mathrm{Cl}, 1.06-1.26)$ and borderline insignificant with frontal-plus-severe-vertex baldness (OR, 1.14; 95\% Cl, 0.98-1.33).

Conclusions: Subtypes of male pattern baldness at age 45 years were positively associated with colorectal neoplasia. Future studies are warranted to confirm our results and to determine the predictive value of male pattern baldness to identify those at high risk for colorectal neoplasia.

Male pattern baldness (also known as androgenic alopecia) is the most common type of hair loss in men. Affecting 50\% of men above 40 years of age (Randall, 2010), it is caused by interplay of genetic predisposition, hormones, and lifestyle factors including smoking, alcohol drinking and stress (Randall, 2010; Gatherwright et al, 2013). From a hormonal perspective, the primary contributor is dihydrotestosterone (DHT) (Randall, 2010). When circulating testosterone enters hair follicles, particularly balding hair follicles, the enzyme $5 \alpha$-reductase metabolises testosterone to biologically more potent DHT that strongly binds to the androgen receptor and alters gene expression, leading hair follicles to shorten their growth phase and to miniaturise (Randall, 2010). Finasteride, a commonly

\footnotetext{
*Correspondence: Dr N Keum; E-mail: nak212@mail.harvard.edu

${ }^{8}$ These authors contributed equally to this work.
}

Received 7 October 2015; revised 15 November 2015; accepted 22 November 2015

(c) 2016 Cancer Research UK. All rights reserved 0007-0920/16 
prescribed drug for male pattern baldness, acts by inhibiting the conversion of testosterone to DHT (Bolduc and Shapiro, 2000). Yet, not only androgens but also insulin-like growth factor 1 (IGF-1) and insulin may be implicated in male pattern baldness, as indicated by men with baldness having elevated levels of circulating IGF-1 (Signorello et al, 1999; Platz et al, 2000) or an increased risk of hyperinsulinaemia/insulin resistance (Trieu and Eslick, 2014).

Testosterone, IGF-1, and insulin are also implicated in the pathogenesis of colorectal neoplasia (cancer (CRC) and its precursory adenoma (CRA)). Although evidence is still limited, androgens may protect against colorectal neoplasia in men through its direct activation of the androgen signalling pathway, leading to reduced expression of oncogenes modulated by $\beta$-catenin or by exerting favourable effects on major risk factors for colorectal neoplasia such as obesity and insulin resistance (Lin and Giovannucci, 2010). In line with this hypothesis, previous studies reported an inverse association between circulating testosterone and the risk of colorectal cancer in men (Holland et al, 1993; Lin et al, 2013). Bioavailable IGF-1 increases proliferation and decreases apoptosis of colonocytes, thereby promoting colorectal carcinogenesis (Giovannucci, 2001; Sandhu et al, 2002). Insulin, apart from its direct mitogenic effect on neoplastic cells, also acts by enhancing bioavailability of IGF-1 through suppressing hepatic production of hormonal binding proteins including IGFBP-1 (Giovannucci et al, 2010). Insulin-like growth factor 1 exerts more potent growth-promoting and antiapoptotic effects than insulin (Giovannucci et al, 2010). Therefore, it is biologically plausible that male pattern baldness, as a marker of underlying aberration in the regulation of androgens, IGF-1, and/or insulin, may be associated with colorectal neoplasia. Yet, no epidemiologic study has evaluated the relationship. Thus, we prospectively examined male pattern baldness at age 45 years in relation to risks of CRC and CRA in the Health Professionals Follow-Up Study (HPFS).

\section{MATERIALS AND METHODS}

Study population. The HPFS began in 1986, enrolling 51529 male health professionals in the United States between 40 and 75 years of age. Participants completed a questionnaire on demographics, medical history, and lifestyle factors at enrolment and have updated information through biennial follow-up questionnaires. They also reported dietary intake through a validated semiquantitative food questionnaire (Rimm et al, 1992; Feskanich et al, 1993) at enrolment and every 4 years thereafter. Follow-up rates have exceeded $90 \%$ in each 2 -year cycle.

For this analysis, the baseline year was defined as 1992 when information on hair pattern at age 45 years was collected. At baseline, the mean age of participants was 60.5 years (s.d. of 9.8 years). We excluded men with a prior diagnosis of CRC or polyp (adenoma analysis only), those who left the hair pattern question blank, and those who developed ulcerative colitis before baseline or over the follow-up. Of note, because of the asymptomatic nature of adenomas, adenomas are generally detected during endoscopic procedures for screening or unrelated gastrointestinal conditions. To minimise potential for detection bias induced by differential frequency of endoscopy across hair patterns, we restricted adenoma analysis to those who underwent at least one colonoscopy or sigmoidoscopy between 1992 and 2010. After these exclusions, 32782 men for CRC analysis and 29770 men for adenoma analysis remained eligible for the follow-up from 1992 through 2010.

Assessment of exposure. The 1992 questionnaire asked participants to select their hair pattern at age 45 years from the five images (no baldness, frontal-only baldness, frontal-plus-mild-vertex baldness, frontal-plus-moderate-vertex baldness, and frontal-plus-

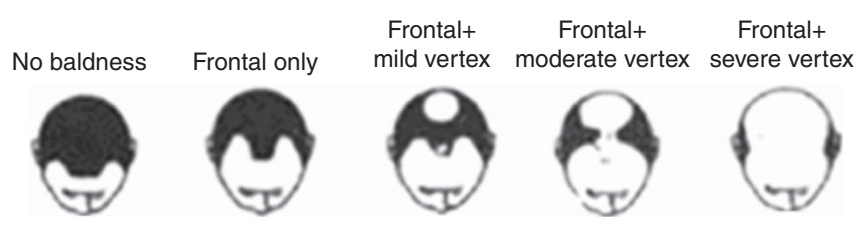

Figure 1. Pictograms of male hair patterns used in the questionnaire.

severe-vertex baldness) modified from the Hamilton-Norwood scale (Figure 1; Norwood, 1975). Of the five hair categories, the four baldness patterns were collectively termed as any baldness in this study.

Assessment of covariates. Information on potential confounders and intermediates, determined a priori from known or suspected risk factors for colorectal neoplasia, was collected at baseline and throughout follow-up. The following lists the definitions of the covariates used for this study: age (years in continuous for cancer analyses; <64.9, 65-69.9, 70-74.9, 75-79.9, $\geqslant 80$ years for adenoma analyses), race (White $v s$ nonwhite), body mass index ( $\mathrm{kg} \mathrm{m}^{-2}$ in quintiles), physical activity (metabolic equivalent task (MET)-hours per week in quintiles), personal history of diabetes mellitus (yes vs no), personal history of endoscopy and polyp detection (no endoscopy + no polyp detection, endoscopy + no polyp detection, endoscopy + polyp detection), time period of endoscopy (integer indicating questionnaire cycles in continuous), number of endoscopies received (continuous), time since the most recent endoscopy (years in continuous), reason for the current endoscopy (screening vs symptoms), family history of colon cancer (yes $v s$ no), smoking habits (never smoker, 0.1-4.9, 5-19.9, 20-39.9, $40+$ pack-years), aspirin use (yes vs no), multivitamin use (yes $v s$ no), finasteride use (yes $v s$ no), total calorie intake (kcal day $^{-1}$ in quintiles), alcohol intake (0, 0.1-4.9, 5.0-14.9, $\geqslant 15 \mathrm{~g} \mathrm{day}^{-1}$ ), red and processed meat intake (quintiles in servings per day), folate intake ( $\mu \mathrm{g} \mathrm{day}^{-1}$ in quintiles), calcium intake (mg day ${ }^{-1}$ in quintiles), and vitamin $\mathrm{D}$ intake (IU day ${ }^{-1}$ in quintiles).

Of note, micronutrient intakes included both dietary and supplemental sources. Covariates with missing values were handled via carry-forward or missing category (i.e., smoking habits, reason for the current endoscopy), but the proportion of participants with missing data was low for most covariates.

Ascertainment of outcome. The case was defined as primary CRC restricted to invasive adenocarcinoma for cancer analysis; as adenomatous polyps for adenoma analysis. On biennial follow-up questionnaires through 2010, participants reported whether they were diagnosed with CRC or polyps. When a diagnosis was reported, after obtaining consent, study investigators blinded to the participants' exposure status reviewed medical records to confirm the diagnosis and to extract information on tumour characteristics including anatomic location, histologic type, and size and number of polyps. Only confirmed cases were included in this analysis.

Statistical analysis. For cancer analysis, hazard ratios (HRs) and 95\% confidence intervals (CIs) for the association between hair pattern at age 45 years and CRC risk were estimated based on Cox proportional hazards models using age as the underlying timescale. Following the Anderson-Gill data structure (Therneau, 1997), a new record was created in each questionnaire cycle for participants at risk by assigning covariates the value of the cycle before the current cycle. Person-time of follow-up was accrued from the date of return of the baseline questionnaire until the date of CRC diagnosis, date of death from any cause, or date of end of study (31 January 2010 for HPFS), whichever came first. The proportional hazard assumption regarding the exposure variable was verified by likelihood ratio test comparing the final multivariable 
model with and without four cross-product terms between categorical exposure and binary age $(<65, \geqslant 65$ years).

For adenoma analysis, the Anderson-Gill data structure was used (Therneau, 1997), where a new data record was created each time men reported endoscopy at biennial follow-up questionnaires by assigning covariates the value of the cycle before the most recent endoscopy. When participants had multiple endoscopies during the follow-up, they contributed multiple data points until the endoscopy when the first adenoma was detected or the last endoscopy done (i.e., person-endoscopy at risk). To account for the correlation over repeated endoscopies for the same individual, logistic regressions for clustered data (SAS PROC GENMOD) was used to estimate odds ratios (ORs) and $95 \%$ CIs.

In multivariable analyses for colorectal neoplasia, potential confounders were adjusted for by updating their values whenever new information was obtained from the follow-up questionnaires. Time-invariant variables (race, family history of colon cancer) and variables with evidence of a long induction period to affect colorectal neoplasia (calcium (Keum et al, 2014, 2015), folate (Lee et al, 2011; Keum and Giovannucci, 2014), and aspirin (Giovannucci et al, 1995; Chan et al, 2005)) were adjusted for using baseline values. Of note, as finasteride use may lie along the causal pathway between male hair patterns and colorectal neoplasia, multivariable analyses were run without and with adjustment for finasteride use.
Colorectal neoplasia is a biologically heterogeneous disease that develops through distinctive aetiologic pathways (Greystoke and Mullamitha, 2012). To explore potential aetiologic heterogeneity in the relationships between hair pattern at age 45 years and colorectal neoplasia, we conducted subgroup analyses by subtypes of colorectal neoplasia defined by tumour characteristics including anatomic location (distal colon, proximal colon, rectum). We classified adenomas by histology ((tubulo)villous vs tubular); by size (large: $\geqslant 1 \mathrm{~cm} v s$ small: $<1 \mathrm{~cm}$ in diameter); by multiplicity $(\geqslant 3$ vs 1-2 ); by risk of progressing to CRC as indicated by the combined characteristics of histology, size, and multiplicity (highrisk: (tubulo)villous or high-grade dysplasia, large, or $\geqslant 3$ adenomas) vs low-risk: tubular, small, and 1-2 adenomas). Furthermore, in light of considerable distinction in the profile of genetic mutations between tubular and villous adenomas (Vogelstein et al, 1988; Yamada et al, 2012), we also examined heterogeneity by subdividing adenomas of large or $\geqslant 3$ by histologic origin (large or $\geqslant 3$ adenomas with (tubulo)villous origin $v s$ large or $\geqslant 3$ adenomas with tubular origin).

Of note, sigmoidoscopy cannot detect proximal colon adenoma, potentially leading to outcome misclassification. Thus, for adenoma analysis, a sensitivity analysis was conducted by restricting the analytic cohort to men who had at least one colonoscopy. For participants with more than one adenoma diagnosed, analyses were performed using the adenoma of the largest size and most advanced histologic characteristics.

Table 1. Characteristics of person-years or endoscopy-years over 1992-2010 by male pattern baldness at age 45 years in HPFS

\begin{tabular}{|c|c|c|c|c|c|}
\hline \multirow[b]{2}{*}{ Characteristics } & \multicolumn{5}{|c|}{ Male pattern baldness } \\
\hline & $\begin{array}{c}\text { No } \\
\text { baldness }\end{array}$ & $\begin{array}{l}\text { Frontal } \\
\text { only }\end{array}$ & $\begin{array}{c}\text { Frontal }+ \text { mild } \\
\text { vertex }\end{array}$ & $\begin{array}{c}\text { Frontal }+ \text { moderate } \\
\text { vertex }\end{array}$ & $\begin{array}{c}\text { Frontal + severe } \\
\text { vertex }\end{array}$ \\
\hline \multicolumn{6}{|l|}{ Colorectal cancer analysis } \\
\hline No. of person-years & 224070 & 131004 & 86103 & 40528 & 28802 \\
\hline Age (year) & $66.7(10.2)$ & $66.9(10.3)$ & $66.1(10.2)$ & $67.4(10.4)$ & $66.8(10.4)$ \\
\hline Caucasian (\%) & 90.6 & 91.7 & 91.4 & 90.8 & 92.7 \\
\hline $\mathrm{BMI}\left(\mathrm{kg} \mathrm{m}^{-2}\right)$ & $25.7(3.2)$ & $25.6(3.1)$ & $25.8(3.2)$ & $25.9(3.5)$ & $26.0(3.3)$ \\
\hline Physical activity (MET-hours/week) & $33.8(28.0)$ & $33.4(27.0)$ & $32.8(27.9)$ & $33.1(28.2)$ & $32.8(27.8)$ \\
\hline Personal history of diabetes mellitus (\%) & 8.3 & 8.1 & 8.4 & 8.4 & 9.5 \\
\hline Personal history of endoscopy and polyp detection (\%) & 18.6 & 18.6 & 18.9 & 18.1 & 19.4 \\
\hline Family history of colon cancer (\%) & 16.2 & 15.6 & 16.7 & 15.0 & 15.1 \\
\hline Pack-years among ever smokers (pack-years) & $26.1(20.4)$ & $25.8(20.3)$ & $25.7(20.8)$ & $25.9(20.1)$ & $26.5(19.7)$ \\
\hline Aspirin use ${ }^{a}(\%)$ & 43.9 & 44.5 & 44.5 & 44.8 & 45.9 \\
\hline Multivitamin use (\%) & 52.7 & 52.2 & 53.0 & 52.9 & 51.1 \\
\hline Finasteride use (\%) & 3.3 & 3.5 & 3.8 & 4.1 & 3.9 \\
\hline Alcohol $\left(\right.$ g day $\left.^{-1}\right)$ & $11.5(13.6)$ & $11.0(13.0)$ & $10.7(13.2)$ & $10.3(12.5)$ & $10.5(13.5)$ \\
\hline Red/processed meat (servings per day) & $1.0(0.7)$ & $1.0(0.7)$ & $1.0(0.7)$ & $1.0(0.7)$ & $1.0(0.7)$ \\
\hline Folate $^{\mathrm{a}}\left(\mu \mathrm{g}_{\text {day }}{ }^{-1}\right)$ & $493(233)$ & $489(224)$ & $490(223$ & $491(230$ & $483(231)$ \\
\hline Calcium ${ }^{\mathbf{a}}\left(\mathrm{mg} \mathrm{day}^{-1}\right)$ & 907 (357) & 906 (352) & $898(343)$ & $900(363)$ & $892(351)$ \\
\hline Vitamin D (IU day $\left.{ }^{-1}\right)$ & $441(238)$ & $437(229)$ & $445(234)$ & $437(232)$ & 435 (239) \\
\hline \multicolumn{6}{|l|}{ Colorectal adenoma analysis } \\
\hline No. of person-endoscopies & 24946 & 14459 & 9993 & 4476 & 3283 \\
\hline Age (year) & $65.8(8.8)$ & $66.0(8.9)$ & $65.4(8.7)$ & $66.3(9.0)$ & $65.7(9.0)$ \\
\hline Caucasian (\%) & 90.4 & 91.5 & 91.6 & 89.2 & 92.0 \\
\hline $\mathrm{BMI}\left(\mathrm{kg} \mathrm{m}^{-2}\right)$ & $25.7(3.1)$ & $25.5(3.0)$ & $25.8(3.1)$ & $25.8(3.2)$ & $26.1(3.4)$ \\
\hline Physical activity (MET-hours/week) & $35.0(27.6)$ & $35.7(27.4)$ & $34.3(26.8)$ & $34.9(28.1)$ & $33.6(27.6)$ \\
\hline Personal history of diabetes mellitus (\%) & 7.3 & 7.5 & 7.1 & 7.4 & 8.7 \\
\hline Family history of colon cancer (\%) & 18.1 & 17.2 & 17.3 & 16.6 & 17.4 \\
\hline Pack-years among ever smokers (pack-years) & $23.6(19.0)$ & $23.3(18.9)$ & 22.5 (18.9) & $23.3(18.6)$ & $25.3(18.0)$ \\
\hline Aspirin use ${ }^{a}(\%)$ & 44.7 & 45.1 & 45.9 & 46.5 & 47.1 \\
\hline Multivitamin use (\%) & 58.4 & 57.9 & 58.9 & 58.4 & 58.1 \\
\hline Finasteride use (\%) & 3.3 & 3.6 & 4.0 & 5.0 & 3.9 \\
\hline Alcohol (g day ${ }^{-1}$ ) & $11.3(12.8)$ & $10.9(12.1)$ & $10.9(12.4)$ & $10.4(11.9)$ & 10.5 (12.9) \\
\hline Red/processed meat (servings per day) & $1.0(0.7)$ & $1.0(0.7)$ & $0.9(0.7)$ & $0.9(0.6)$ & $0.9(0.7)$ \\
\hline Folate $^{\mathbf{a}}\left(\mu \mathrm{g} \mathrm{day}^{-1}\right)$ & $497(230)$ & $493(220)$ & $496(224)$ & 499 (239) & $501(243)$ \\
\hline Calcium ${ }^{\mathbf{a}}\left(\mathrm{mg} \mathrm{day}^{-1}\right)$ & 905 (353) & $906(335)$ & $906(343)$ & $893(348)$ & 902 (349) \\
\hline Vitamin D (IU day $\left.{ }^{-1}\right)$ & $448(233)$ & $447(221)$ & $457(231)$ & $447(233)$ & $451(234)$ \\
\hline
\end{tabular}


All the statistical tests were two sided and $P$-values of $\leqslant 0.05$ were considered statistically significant. Analyses were performed using SAS 9.3 (SAS Institute, Cary, NC, USA).

\section{RESULTS}

We documented 710 incident cases of CRC among 32782 eligible men over the mean follow-up of 15.6 years (510 507 person-years); 3526 cases of newly diagnosed CRA among 29770 men who underwent at least one endoscopy between 1992 and 2010 (57 157 person-endoscopies). Across the analytic cohorts (Table 1), men with frontal-plus-severe-vertex baldness at age 45 years were more likely to have diabetes mellitus and to use aspirin and finasteride than men without baldness at age 45 years. In addition, within the analytic cohort for adenoma analysis, men with frontalplus-severe-vertex baldness tended to engage in less physical activity and to have smoked more cigarettes. These differences tended to be slight, and other potential risk factors for colorectal neoplasia were distributed similarly across the different hair patterns. When the two analytic cohorts were compared, men in the adenoma analysis were less likely to be diabetic and more likely to be physically active; to have a family history of colon cancer; and to use multivitamin.

In the cancer analyses in relation to hair pattern at age 45 years (Table 2), evidence for an increased risk associated with any baldness compared with no baldness was suggestive for overall CRC (HR, 1.13; 95\% CI, 0.97-1.32), but significant for colon cancer (HR, 1.24; 95\% CI, 1.02-1.50), particularly proximal colon cancer (HR, 1.30; 95\% CI, 1.00-1.68). Analyses by subtypes of baldness showed that the positive association was largely driven by frontal-only baldness and frontal-plus-mild-vertex baldness. Compared with men with no baldness, the risk of colon cancer increased by $29 \%$ for men with frontal-only baldness (HR, 1.29; 95\% CI, $1.03-1.62$ ) and by $31 \%$ for men with frontal-plus-mildvertex baldness (HR, 1.31; 95\% CI, 1.01-1.70). For proximal colon cancer, the HR comparing frontal-plus-mild-vertex baldness and no baldness amounted to 1.42 (95\% CI, 1.00-2.02). Further adjustment for finasteride use barely changed the results (data not shown).

In the adenoma analyses in relation to hair pattern at age 45 years (Table 3), evidence for a positive association was diluted when analysed with composite exposure of any baldness, but became evident with some subtypes of baldness. Compared with no baldness, a statistically significant increased risk of CRA was observed for frontal-only baldness (OR, 1.16, 95\% CI, $1.06-1.26$ ) and a borderline-insignificant positive association was observed for frontal-plus-severe-vertex baldness (OR, 1.14, 95\% CI, 0.98-1.33). By anatomic subsites, the association with frontal-only baldness appeared stronger for distal colon and rectal adenomas, whereas that with frontal-plus-severe-vertex baldness was pronounced in the proximal colon and rectum adenomas. Upon restricting the analysis to men who had at least one colonoscopy, the results did not change materially except that the aforementioned associations became slightly stronger (data not shown); an association of rectal adenoma with frontal-only baldness became significant $(\mathrm{OR}, 1.30$, 95\% CI, 1.03-1.63, $P=0.03$ ).

The relationship between subtypes of hair pattern at age 45 years and adenoma was also heterogeneous by characteristics

Table 2. $\mathrm{HR}$ and $95 \% \mathrm{Cl}$ for male pattern baldness at age 45 years and total and site-specific colorectal cancer

\begin{tabular}{|c|c|c|c|c|c|c|}
\hline \multirow[b]{2}{*}{ Anatomic site } & \multicolumn{6}{|c|}{ Male pattern baldness } \\
\hline & No baldness & Frontal only & $\begin{array}{c}\text { Frontal }+ \text { mild } \\
\text { vertex }\end{array}$ & $\begin{array}{c}\text { Frontal }+ \text { moderate } \\
\text { vertex }\end{array}$ & $\begin{array}{c}\text { Frontal + severe } \\
\text { vertex }\end{array}$ & Any baldness \\
\hline \multicolumn{7}{|l|}{ Colorectal cancer } \\
\hline No. of cases & 285 & 195 & 133 & 57 & 40 & 425 \\
\hline Age adjusted ${ }^{a}$ & 1 (Referent) & $1.16(0.96-1.39)$ & $1.23(1.00-1.52)$ & $1.02(0.76-1.36)$ & $1.11(0.79-1.55)$ & $1.15(0.99-1.34)$ \\
\hline Multivariable ${ }^{\mathbf{b}}$ & 1 (Referent) & $1.13(0.94-1.36)$ & $1.22(0.98-1.50)$ & $0.99(0.74-1.33)$ & $1.09(0.78-1.53)$ & $1.13(0.97-1.32)$ \\
\hline$P$-value for multivariable analysis & Referent & 0.19 & 0.07 & 0.96 & 0.62 & 0.12 \\
\hline \multicolumn{7}{|l|}{ Colon cancer } \\
\hline No. of cases & 182 & 140 & 92 & 37 & 27 & 296 \\
\hline Age adjusted ${ }^{a}$ & 1 (Referent) & $1.32(1.05-1.65)$ & $1.33(1.03-1.71)$ & $1.02(0.71-1.46)$ & $1.16(0.77-1.75)$ & $1.26(1.04-1.52)$ \\
\hline Multivariable ${ }^{\mathbf{b}}$ & 1 (Referent) & $1.29(1.03-1.62)$ & $1.31(1.01-1.70)$ & $1.01(0.70-1.45)$ & $1.14(0.75-1.72)$ & $1.24(1.02-1.50)$ \\
\hline$P$-value for multivariable analysis & Referent & 0.02 & 0.04 & 0.96 & 0.55 & 0.03 \\
\hline \multicolumn{7}{|l|}{ Proximal colon cancer } \\
\hline No. of cases & 95 & 76 & 50 & 21 & 12 & 159 \\
\hline Age adjusted ${ }^{\mathbf{a}}$ & 1 (Referent) & 1.37 (1.01-1.86) & $1.43(1.01-2.03)$ & $1.09(0.67-1.75)$ & $0.97(0.53-1.79)$ & 1.31 (1.01-1.69) \\
\hline Multivariable ${ }^{\mathbf{b}}$ & 1 (Referent) & $1.34(0.99-1.83)$ & $1.42(1.00-2.02)$ & $1.11(0.68-1.80)$ & $0.98(0.53-1.81)$ & 1.30 (1.00-1.68) \\
\hline$P$-value for multivariable analysis & Referent & 0.06 & 0.05 & 0.68 & 0.95 & 0.05 \\
\hline \multicolumn{7}{|l|}{ Distal colon cancer } \\
\hline No. of cases & 81 & 60 & 34 & 16 & 13 & 123 \\
\hline Age adjusted $^{a}$ & 1 (Referent) & $1.28(0.91-1.80)$ & $1.06(0.71-1.60)$ & 1.01 (0.59-1.74) & $1.31(0.72-2.37)$ & $1.17(0.88-1.56)$ \\
\hline Multivariable $\mathbf{b}$ & 1 (Referent) & $1.24(0.88-1.75)$ & $1.03(0.68-1.56)$ & $0.97(0.56-1.68)$ & $1.26(0.69-2.32)$ & $1.14(0.85-1.51)$ \\
\hline$P$-value for multivariable analysis & Referent & 0.22 & 0.89 & 0.91 & 0.45 & 0.39 \\
\hline \multicolumn{7}{|l|}{ Rectal cancer } \\
\hline No. of cases & 64 & 41 & 24 & 14 & 9 & 88 \\
\hline Age adjusted ${ }^{a}$ & 1 (Referent) & $1.04(0.70-1.55)$ & $0.99(0.61-1.61)$ & $1.19(0.66-2.14)$ & $1.10(0.54-2.22)$ & $1.05(0.76-1.46)$ \\
\hline Multivariable & 1 (Referent) & $1.05(0.70-1.57)$ & $1.04(0.64-1.69)$ & $1.24(0.68-2.25)$ & $1.05(0.50-2.17)$ & $1.08(0.77-1.50)$ \\
\hline$P$-value for multivariable analysis & Referent & 0.81 & 0.88 & 0.47 & 0.90 & 0.67 \\
\hline \multicolumn{7}{|c|}{ 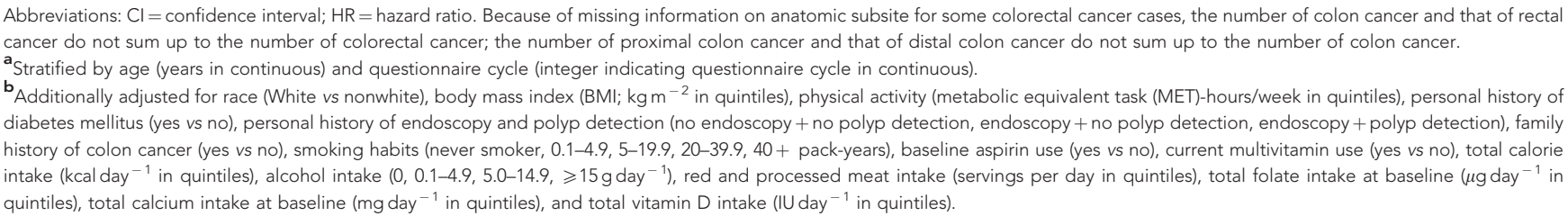 } \\
\hline
\end{tabular}


Table 3. OR and $95 \% \mathrm{Cl}$ for male pattern baldness at age 45 years and total and site-specific colorectal adenoma

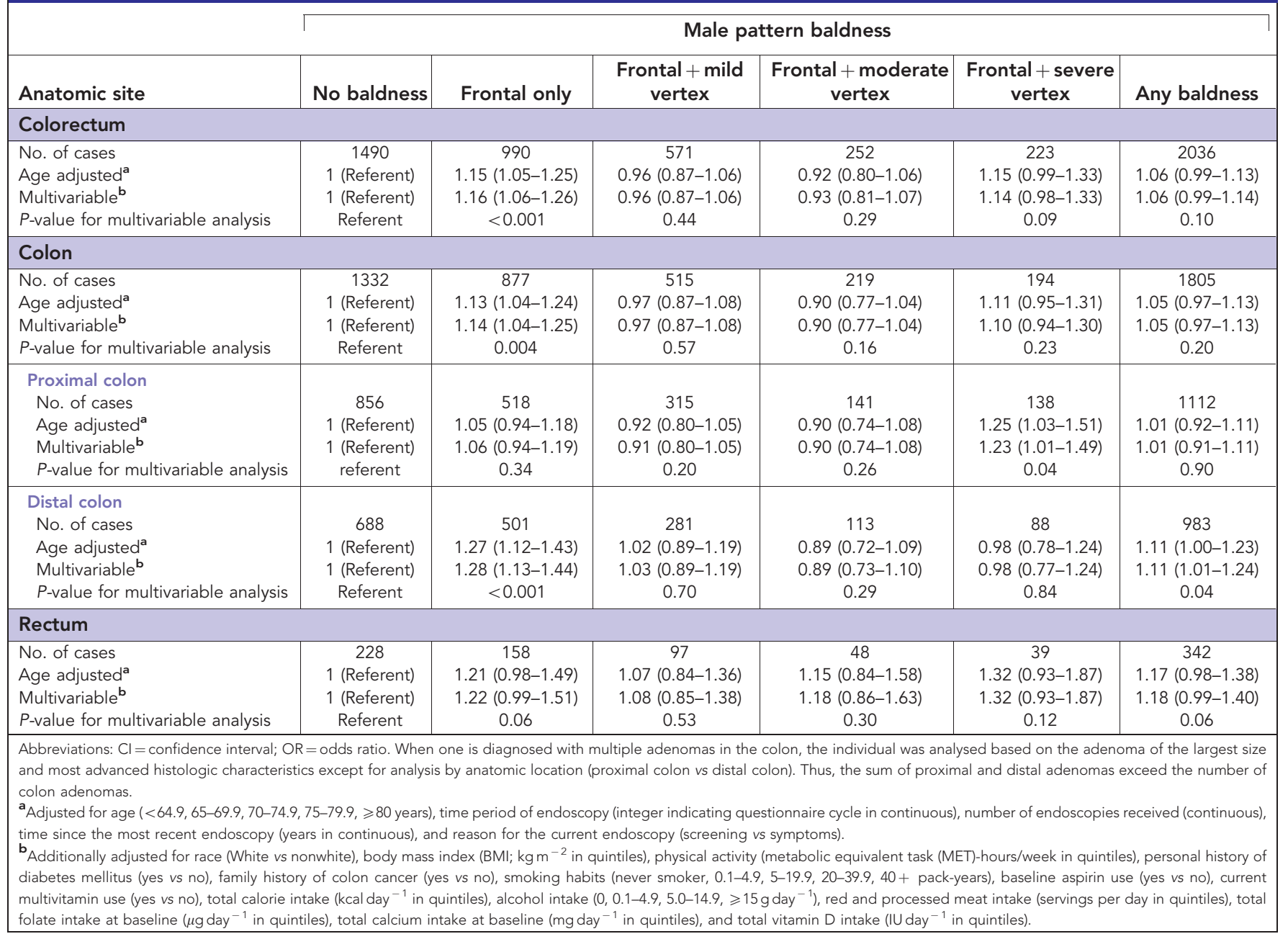

related to histopathology, size, and multiplicity (Table 4). An elevated risk associated with frontal-only baldness and frontalplus-severe-vertex baldness was primarily with tubular adenoma, small adenoma, 1-2 adenomas, and low-risk adenoma. When adenomas of large size or with multiplicity $(\geqslant 3)$ were further stratified by histopathology, significant evidence of an increased risk associated with frontal-only baldness was observed for adenomas with tubular histopathology but not for adenomas with (tubulo)villous histopathology.

\section{DISCUSSION}

Among men, some patterns of baldness at age 45 years were significantly associated with an increased risk of colorectal neoplasia compared with no baldness. For cancer, frontal-only baldness and frontal-plus-mild-vertex baldness were positively associated with colon cancer but not with rectal cancer. For adenomas, an elevated risk of colon adenoma was also observed for frontal-only baldness but not with frontal-plus-mild-vertex baldness. In addition for rectal adenoma, suggestive nonsignificant positive associations were observed with frontal-only baldness and with frontal-plus-severe-vertex baldness. By subtypes of adenomas, these associations were largely confined to adenomas with tubular histopathology. Although a significant association was not observed for large or multiple adenomas overall, frontal-only baldness formed a significant positive association with large or multiple adenomas of tubular histology. Finasteride use had little influence on the results.

Previous studies on male pattern baldness and malignancy focused on prostate cancer because of its androgen dependency. In a recent prospective cohort study conducted among participants in the Prostate, Lung, Colorectal, and Ovarian Cancer Screening Trial, it was only frontal-plus-moderate-vertex baldness at age 45 years that was associated with an increased risk of aggressive prostate cancer (Zhou et al, 2015). This result juxtaposes our finding that it was only frontal-plusmoderate-vertex baldness at age 45 years that was not associated with any colorectal neoplasia. While the main hypothesis proposed to explain a positive association between baldness and prostate cancer relates to excess androgenicity (Craft and Sawyers, 1998), for CRC development in men, high testosterone does not increase risk and may even be protective (Lin and Giovannucci, 2010). Thus, the type of baldness linked to an elevated risk of aggressive prostate cancer is not expected to be associated with an increased risk of colorectal neoplasia. Furthermore, evidence supporting elevated levels of circulating testosterone among bald men remains inconclusive (Randall, 2010). Some studies observed normal levels of circulating testosterone among bald men (Phillipou and Kirk, 1981; Pitts, 1987), suggesting that responsiveness to circulating testosterone of hair follicles as determined by intracellular androgen receptor number and $5 \alpha$-reductase activity may be more relevant to inducing male pattern baldness than circulating testosterone concentration per se. 
Table 4. OR and $95 \% \mathrm{Cl}$ for male pattern baldness at age 45 years and colorectal adenoma by subtypes

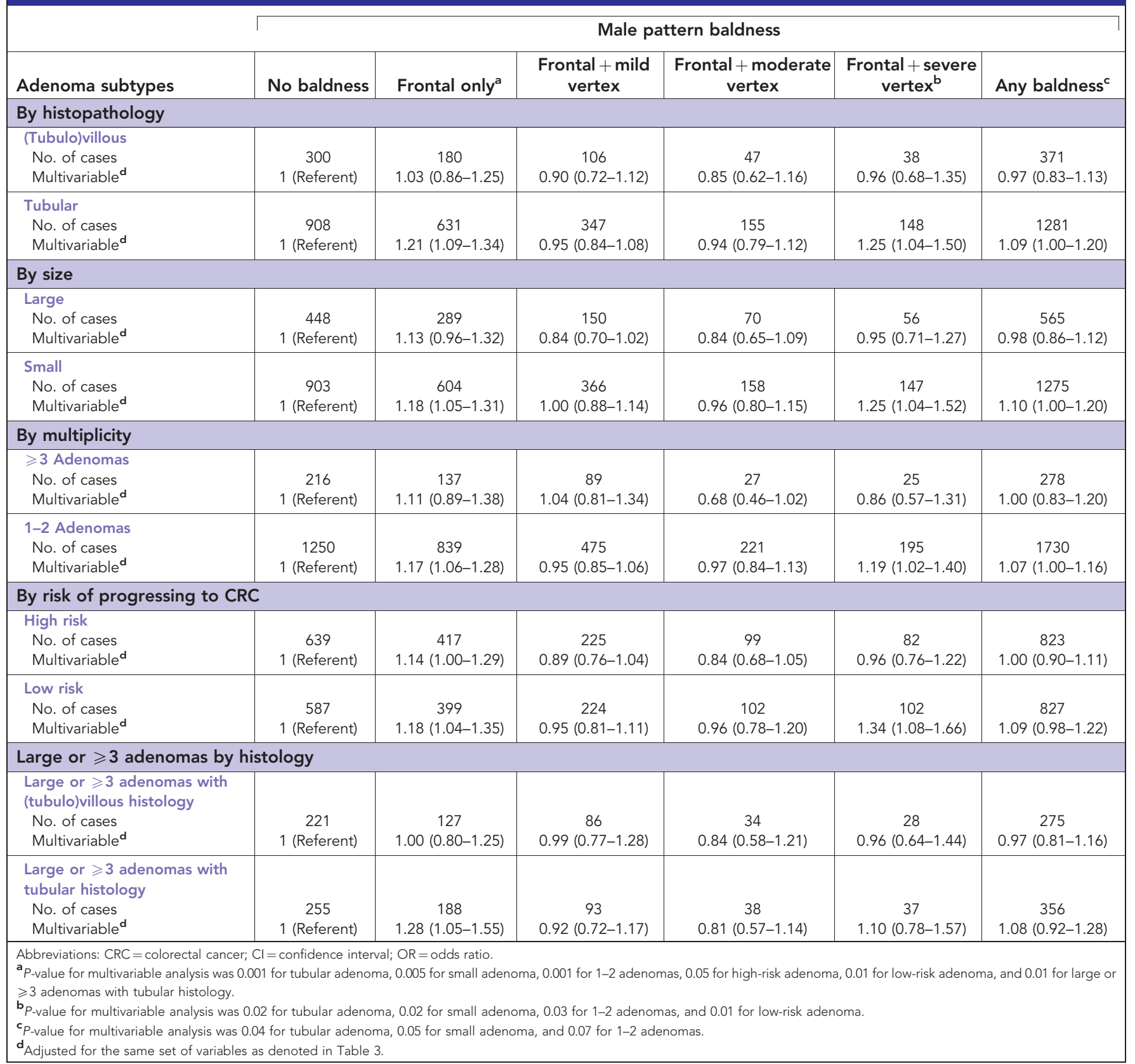

Rather than testosterone, we hypothesise that the IGF-1 and insulin pathways may be factors linking male pattern baldness to colorectal neoplasia (particularly CRC). In a cross-sectional study that examined mutually adjusted levels of circulating testosterone, IGF-1, and other sex hormones and their binding proteins, only IGF-1 was significantly associated with an increased risk of baldness (Signorello et al, 1999). Moreover, a recent meta-analysis found that baldness was associated with an increased risk of insulin resistance (pooled OR, 4.88; 95\% CI, 2.05-11.64) and hyperinsulinaemia (pooled OR, 1.97; 95\% CI, 1.20-3.21) (Trieu and Eslick, 2014). In our study, an increased risk associated with baldness was virtually confined to adenomas of small size, tubular histology, and/or 1-2 adenomas. Interestingly, in a recent study that examined insulin-related serum markers in relation to types of CRA in Caucasian males, circulating C-peptide, a marker of insulin secretion, was strongly associated with tubular adenomas (OR comparing extreme tertiles, 3.8; 95\% CI, 1.3-11.2)
(Comstock et al, 2014). Thus, the insulin pathway may be particularly relevant to linking baldness to tubular adenomas and, indeed in our study, a positive association was suggested for large or multiple adenomas if they are of tubular histology.

It is worth noting that an increased risk associated with frontalplus-severe-vertex baldness was evident for CRA but not for CRC. The role of chance cannot be excluded. Yet, as for the association with frontal-only baldness, the association with frontal-plussevere-vertex baldness was confined to adenomas of tubular histology. Although lack of studies on hormone profiles across each type of baldness hampers our understanding of biological mechanism underlying the heterogeneous finding, in light of an estimated $79 \%$ heritability of male pattern baldness (Rexbye et al, 2005), reaching the most progressed type of baldness at age 45 years may be driven by a strong genetic influence. Thus, it is plausible that there are genetic polymorphisms common to severe baldness and adenomas of tubular histology. 
Our finding that male pattern baldness was associated with risk of tubular but not (tubulo)villous adenoma may suggest some aetiologic insights. Tubulo-villous and villous adenomas are more likely to progress and be of advanced stage than tubular adenomas, and this may explain why baldness was weakly associated with overall high-risk adenomas. However, baldness was strongly and significantly associated with the subset of high-risk adenomas that had exclusively a tubular component.

There are several strengths in our study. To our knowledge, our study represents the first to report a potential relationship between male pattern baldness and the risk of colorectal neoplasia. By examining both adenoma and cancer outcomes and observing a positive association across neoplasias in colorectal carcinogenesis, we reduced the likelihood of chance findings. Furthermore, we had little evidence of confounding, as indicated by minimal association between baldness and lifestyle factors and little change in results from the age-adjusted to multivariable-adjusted model. By analysing by subtypes of colorectal neoplasia and accounting for finasteride use, our study was able to elucidate some mechanistic insight regarding the role of hormones across colorectal carcinogenesis.

Yet, our study has limitations. First, male pattern baldness at age 45 years was recalled and self-reported. Aside from random errors associated with recall, as baldness is considered unattractive, participants may have downgraded the severity of baldness. However, as this information was assessed before the diagnosis of colorectal neoplasia, misclassification is likely to be random with respect to the outcome, which can bias results toward the null. Yet, we were still able to observe significant associations. Second, our cancer analyses may have inadequate power to examine an association with more progressed types of baldness because of small number of CRC cases for frontal-plus-moderate-vertex baldness and frontal-plus-severe-vertex baldness. Yet, we attempted to address the limitation by complementing the cancer analyses with the adenoma analyses having approximately five times as many cases. Third, as male pattern baldness is a complex disorder determined by interplay of genetic and nongenetic factors, baldness pattern at different ages or rate of progressiveness may better capture the underlying factor responsible for an increased risk of colorectal neoplasia. However, our study with a single measurement of hair pattern could not address these questions. Finally, our findings may not be generalisable to other racial populations.

In conclusion, subtypes of male pattern baldness at age 45 years were positively associated with colorectal adenoma and cancer. To determine the predictive value of male pattern baldness in identifying those at high risk for colorectal neoplasia, our results need to be confirmed in future large epidemiologic studies. To better understand aetiologic mechanism linking male pattern baldness to colorectal neoplasia, additional studies are warranted to examine the association of sex hormones, IGFs, and insulin in relation to subtypes of male pattern baldness.

\section{ACKNOWLEDGEMENTS}

$\mathrm{NaNa}$ Keum had full access to all of the data in the study and takes responsibility for the integrity of the data and the accuracy of the data analysis. We thank the participants and staff of the HSPF for their valuable contributions as well as the following state cancer registries for their help: $\mathrm{AL}, \mathrm{AZ}, \mathrm{AR}, \mathrm{CA}, \mathrm{CO}, \mathrm{CT}, \mathrm{DE}, \mathrm{FL}, \mathrm{GA}, \mathrm{ID}$, IL, IN, IA, KY, LA, ME, MD, MA, MI, NE, NH, NJ, NY, NC, ND, $\mathrm{OH}, \mathrm{OK}, \mathrm{OR}, \mathrm{PA}, \mathrm{RI}, \mathrm{SC}, \mathrm{TN}, \mathrm{TX}, \mathrm{VA}, \mathrm{WA}$, and WY. This work was supported by the National Institutes of Health (UM1 CA167552).

\section{CONFLICT OF INTEREST}

The authors declare no conflict of interest.

\section{REFERENCES}

Bolduc C, Shapiro J (2000) Management of androgenetic alopecia. Am J Clin Dermatol 1(3): 151-158.

Chan AT, Giovannucci EL, Meyerhardt JA, Schernhammer ES, Curhan GC, Fuchs CS (2005) Long-term use of aspirin and nonsteroidal antiinflammatory drugs and risk of colorectal cancer. JAMA 294(8): 914-923.

Comstock SS, Xu D, Hortos K, Kovan B, McCaskey S, Pathak DR, Fenton JI (2014) Association of insulin-related serum factors with colorectal polyp number and type in adult males. Cancer Epidemiol Biomarkers Prev 23(9): 1843-1851.

Craft N, Sawyers CL (1998) Mechanistic concepts in androgen-dependence of prostate cancer. Cancer Metastasis Rev 17(4): 421-427.

Feskanich D, Rimm EB, Giovannucci EL, Colditz GA, Stampfer MJ, Litin LB, Willett WC (1993) Reproducibility and validity of food intake measurements from a semiquantitative food frequency questionnaire. J Am Diet Assoc 93(7): 790-796.

Gatherwright J, Liu MT, Amirlak B, Gliniak C, Totonchi A, Guyuron B (2013) The contribution of endogenous and exogenous factors to male alopecia: a study of identical twins. Plast Reconstr Surg 131(5): 794e-801e.

Giovannucci E (2001) Insulin, insulin-like growth factors and colon cancer: a review of the evidence. J Nutr 131(11 Suppl): 3109S-3120S.

Giovannucci E, Egan KM, Hunter DJ, Stampfer MJ, Colditz GA, Willett WC, Speizer FE (1995) Aspirin and the risk of colorectal cancer in women. N Engl J Med 333(10): 609-614.

Giovannucci E, Harlan DM, Archer MC, Bergenstal RM, Gapstur SM, Habel LA, Pollak M, Regensteiner JG, Yee D (2010) Diabetes and cancer: a consensus report. Diabetes Care 33(7): 1674-1685.

Greystoke A, Mullamitha SA (2012) How many diseases are colorectal cancer? Gastroenterol Res Pract 2012: 564741.

Holland M, Rotenberg R, Gomez N, Marantz M, D’Angelo N (1993) [Serum testosterone: a possible marker for colorectal cancer]. Medicina (B Aires) 53(2): 117-123.

Keum N, Aune D, Greenwood DC, Ju W, Giovannucci EL (2014) Calcium intake and colorectal cancer risk: dose-response meta-analysis of prospective observational studies. Int J Cancer 135(8): 1940-1948.

Keum N, Giovannucci EL (2014) Folic acid fortification and colorectal cancer risk. Am J Prev Med 46(3 Suppl 1): S65-S72.

Keum N, Lee DH, Greenwood DC, Zhang X, Giovannucci EL (2015) Calcium intake and colorectal adenoma risk: dose-response meta-analysis of prospective observational studies. Int J Cancer 136(7): 1680-1687.

Lee JE, Willett WC, Fuchs CS, Smith-Warner SA, Wu K, Ma J, Giovannucci E (2011) Folate intake and risk of colorectal cancer and adenoma: modification by time. Am J Clin Nutr 93(4): 817-825.

Lin JH, Giovannucci E (2010) Sex hormones and colorectal cancer: what have we learned so far? J Natl Cancer Inst 102(23): 1746-1747.

Lin JH, Zhang SMM, Rexrode KM, Manson JE, Chan AT, Wu KN, Tworoger SS, Hankinson SE, Fuchs C, Gaziano JM, Buring JE, Giovannucci E (2013) Association between sex hormones and colorectal cancer risk in men and women. Clin Gastroenterol Hepatol 11(4): 419-424.e1.

Norwood OT (1975) Male pattern baldness: classification and incidence. South Med J 68(11): 1359-1365.

Phillipou G, Kirk J (1981) Significance of steroid measurements in male pattern alopecia. Clin Exp Dermatol 6(1): 53-56.

Pitts RL (1987) Serum elevation of dehydroepiandrosterone sulfate associated with male pattern baldness in young men. J Am Acad Dermatol 16(3 Pt 1): 571-573.

Platz EA, Pollak MN, Willett WC, Giovannucci E (2000) Vertex balding, plasma insulin-like growth factor 1, and insulin-like growth factor binding protein 3. J Am Acad Dermatol 42(6): 1003-1007.

Randall VA (2010) Molecular basis of androgenetic alopecia. In Aging Hair, Trüeb RM, Tobin DJ (eds), pp 9-24. Springer-Verlag: Berlin Heidelberg.

Rexbye H, Petersen I, Iachina M, Mortensen J, McGue M, Vaupel JW, Christensen K (2005) Hair loss among elderly men: etiology and impact on perceived age. J Gerontol A Biol Sci Med Sci 60(8): 1077-1082. 
Rimm EB, Giovannucci EL, Stampfer MJ, Colditz GA, Litin LB, Willett WC (1992) Reproducibility and validity of an expanded selfadministered semiquantitative food frequency questionnaire among male health professionals. Am J Epidemiol 135(10): 1114-1126; discussion 1127-36.

Sandhu MS, Dunger DB, Giovannucci EL (2002) Insulin, insulin-like growth factor-I (IGF-I), IGF binding proteins, their biologic interactions, and colorectal cancer. J Natl Cancer Inst 94(13): 972-980.

Signorello LB, Wuu J, Hsieh C, Tzonou A, Trichopoulos D, Mantzoros CS (1999) Hormones and hair patterning in men: a role for insulin-like growth factor 1? J Am Acad Dermatol 40(2 Pt 1): 200-203.

Therneau TM (1997) Extending the Cox model. Paper presented at the First Seattle Symposium in Biostatistics: Survival Analysis. Seattle, WA.

Trieu N, Eslick GD (2014) Alopecia and its association with coronary heart disease and cardiovascular risk factors: a meta-analysis. Int J Cardiol 176(3): 687-695.
Vogelstein B, Fearon ER, Hamilton SR, Kern SE, Preisinger AC, Leppert M, Nakamura Y, White R, Smits AM, Bos JL (1988) Genetic alterations during colorectal-tumor development. N Engl J Med 319(9): 525-532.

Yamada M, Sekine S, Ogawa R, Taniguchi H, Kushima R, Tsuda H, Kanai Y (2012) Frequent activating GNAS mutations in villous adenoma of the colorectum. J Pathol 228(1): 113-118.

Zhou CK, Pfeiffer RM, Cleary SD, Hoffman HJ, Levine PH, Chu LW, Hsing AW, Cook MB (2015) Relationship between male pattern baldness and the risk of aggressive prostate cancer: an analysis of the Prostate, Lung, Colorectal, and Ovarian Cancer Screening Trial. J Clin Oncol 33(5): 419-425.

This work is published under the standard license to publish agreement. After 12 months the work will become freely available and the license terms will switch to a Creative Commons AttributionNonCommercial-Share Alike 4.0 Unported License. 